\title{
New carbohydrate-active enzymes identified by screening two metagenomic libraries derived from the soil of a winter \\ wheat field
}

\author{
A. Stroobants ${ }^{1 *}$, D. Portetelle ${ }^{1}$, M. Vandenbol ${ }^{1}$ \\ ${ }^{1}$ Microbiology and Genomics Unit, University of Liège, Gembloux Agro-Bio Tech, Avenue Maréchal \\ Juin 6, 5030 Gembloux, Belgium, email: aurore.stroobants@ulg.ac.be \\ *Corresponding author. Microbiology and Genomics Unit, University of Liège, Gembloux \\ Agro-Bio Tech, Avenue Maréchal Juin 6, 5030 Gembloux, Belgium. Tel.: +32 81622355 . \\ Fax: +32 816215 55. E-mail address: aurore.stroobants@ulg.ac.be.
}

Running headline: New soil CAZymes by metagenomics 


\section{Abstract}

Aim

Soils are rich, diversified environments where $\beta$-glucosidases abound because of their importance in organic matter degradation. The aim of this work was to discover new $\beta$ glucosidases by constructing two metagenomic DNA libraries from soil samples collected in winter and spring from a field of winter wheat.

\section{Methods and Results}

Both libraries were screened on esculin-supplemented medium so as to isolate candidates showing $\beta$-glucosidase activity. Candidate analysis revealed seven putative $\beta$-glycosidases and two putative glycosyltransferases, displaying $25 \%$ to $82 \%$ identity to known enzymes. The putative $\beta$-glycosidases belong to families $\mathrm{GH} 1, \mathrm{GH} 3$, and $\mathrm{GH} 20$ and the two putative glycosyltransferases, probably, to new families. In characterization tests performed on bacteria in suspension or spread on agar plates, some candidates appeared to hydrolyse several natural and synthetic substrates. These tests also highlighted interesting industrial characteristics, such as the activity of four $\beta$-glycosidases under alkaline conditions and the esculin-hydrolysing activity of a $\beta$-glucosidase candidate in the presence of glucose.

\section{Conclusions}

Seven putative $\beta$-glycosidases and two putative glycosyltransferases were found by functional screening of two metagenomic DNA libraries derived from agricultural soil.

\section{Significance and Impact of Study}

This study has identified $\beta$-glycosidases and putative glycosyltransferases that have or may have interesting industrial characteristics.

Keywords: hydrolysing enzyme, functional screening, glucosidase, glycosyltransferase, carbohydrate esterase, soil, metagenomic library, glycoside hydrolase 